\title{
SPEED ADAPTATION IN URBAN ROAD NETWORK MANAGEMENT
}

\author{
Jamal Raiyn \\ Computer Science Department \\ Al Qasemi Academic College \\ Baqa El Gharbia, Israel \\ raiyn@qsm.ac.il
}

\begin{abstract}
Various forecasting schemes have been proposed to manage traffic data, which is collected by videos cameras, sensors, and mobile phone services. However, these are not sufficient for collecting data because of their limited coverage and high costs for installation and maintenance. To overcome the limitations of these tools, we introduce a hybrid scheme based on intelligent transportation system (ITS) and global navigation satellite system (GNSS). Applying the GNSS to calculate travel time has proven efficient in terms of accuracy. In this case, GNSS data is managed to reduce traffic congestion and road accidents. This paper introduces a short-time forecasting model based on real-time travel time for urban heterogeneous road networks. Travel time forecasting has been achieved by predicting travel speeds using an optimized exponential moving Average (EMA) model. Furthermore for speed adaptation in heterogeneous road networks, it is necessary to introduce asuitable control strategy for longitude, based on the GNSS. GNSS products provide worldwide and real-time services using precise timing information and, positioning technologies.
\end{abstract}

Keywords: ITS, EMA, traffic, speed adaptation

\section{Introduction}

Nowadays, protecting human life is an important concern and an economic challenge in regarding to intelligent transportation systems. Heavy traffic in road networks and road accidents has received the most attention from researchers, due their economic impact (Elvik, 2000). Traffic congestion costs the economy billions of dollars. The major cause of traffic congestion is road accidents, which continue to increase in the post- modern age. They have many causes, which can be divided into four categories: (1) the environment, (2) traffic conditions, (3) problems with vehicles, and (4) driver behavior. Many studies have shown that higher speeds do not lead to serious accidents (Tu et. al., 2008). On the other hand, some studies have shown that fatal accidents increase with high speed limits. Our analysis revealed that the major factor leading to an accident is not speed, but variations in speed. To reduction the amount of traffic congestion in road networks, and their negative effects ((i.e. delays, waiting time, driver stress, air and noise pollution, and the blocking of emergency vehicles) and to improve traffic safety, we have introduced a travel data management approach to variations in traffic flow speeds in real-time.

This new form of management is effective and will improve transportation supply performance in time and space with real-time interventions. When the number of vehicles increases in the road networks, high dynamics in traffic flow and increases in travel time follow and traffic management becomes more complex. So we have introduced a road network guide based on the GNNS (Hong et. al., 2014). A mobile agent was designed to handle traffic in heterogeneous road networks by taking into account the real-time traffic flow. The proposed research work in intelligent transportation systems will investigate a novel approach integrating travel observations made by different sources and hybrid applications.

The challenge is to take advantage of each research trend and provide an optimized solution to the intelligent transportation system (ITS). The objective of the proposed management approach is to increase the quality of the entire road network, especially in the case of congestion and jams at peak hours, taking into account real-time traffic information and the travel times of drivers, enabling them to reach their destinations in safety.

Many ITS applications require real-time vehicle positioning data. The main task for a map- matching (MM) algorithm is to identify the correct road segment (Velaga et. al., 2010). A navigation system that provides such positioning data consists of three components:

- a positioning system, such a global positioning system (GPS) (Berzina et. al., 2014),

- a geographic information system (GIS) based road map, and

- a map-matching (MM) algorithm (Quddus et. al., 2007). 
However, a navigation system that provides position data contains errors due to satellite orbits. Errors result from atmospheric effects, receiver measurement error and multipath error (Quddus et. al., 2007).

The mismatching was carried out to identify errors due to:

- positioning sensors,

- $\quad$ digital maps and

- the map-matching process.

Furthermore, GIS-based road maps contain errors that can be geometric or topological (Velaga et. al., 2010). This phenomenon is known as mismatching. Identification of the wrong road link may mislead users and reduce the effectiveness of the ITS service. Current map-matching algorithms have many constraints and limitations, especially in urban areas. Highly accurate positioning data are essential (Quddus et. al., 2007).

This paper is organized as follows: Section 2 presents an overview of related travel data. Section 3 describes the proposed concept for a real-time road supervision model. Section 4 presents a short- time travel forecast model, based on an optimized EMA. Section 5 introduces a map matching method for determining vehicle position. Section 5 presents simulations and results. Finally, the conclusion summarizes the work and points to some directions for future research.

\section{Travel Data Collection}

The objective of the travel time modeling framework was to provide an incident detection algorithm based on historical data, for several purposes. The primary use of historical travel time was to establish the statistical properties of street segment travel times, and thus, make more information available to the incident detection system, in addition to the real-time travel time records provided by the transit probe vehicles. Furthermore, historical data was used to detect outliers in the reported travel times. The development of advanced technologies for data collection and data management has led to the collection of high quality accident data involving numerous types of information, as well as the ability to manage data more efficiently. There are two major strategies for travel data collections. Currently, the most and widely used technology is the traditional strategy based on magnetic loop detectors, installed under the roadway surface (Borzacchielo, 2010).

The lack of this technology is explained by the high cost of installing and maintenance of the local detectors; they are typically installed only on a relatively small area of the roadway system, thus providing limited coverage of the entire transportation network. Furthermore in an urban environment, there are many traffic interruptions that cause delays and that are not easily captured by measuring speeds at any point along the road. To avoid the cost of roadside equipment, we introduce a modern strategy that is based on cellular phone service. Information based on cellular systems can be gathered in milliseconds compared to the traffic data collected from detectors. Conceptually, traffic information may fall into one of the three categories: historical information, real-time information, and predictive information. Historical data consist of past observations of the system. They describe the traffic status of a transportation system during previous time periods, and they mainly used to construct daily graphs or register special events. Real-time information up-to-date and can be calculated, for example, by on-line simulations. Predictive information, such as traffic forecasts, can help to change the travel behavior of road users by providing information about the future state of the network. The real-time information gathered to update the historical adaptive information is considered special when it does not match the historical information. Historical information is needed to carry out a plausible forecast in real-time. To develop a robust forecast model, it is necessary to collect accurate travel information. Firstly, it is necessary to optimize the resource allocation in cellular systems. This involves a consideration of factors like repeated handoffs, radio spectrum coverage, call blocking probability, delays and interference.

Our study and analysis have shown that these factors strongly influence the quality of the collected travel flow information. In the second half of twentieth century, the phenomenon of traffic congestion became prominent, due to a rapid increase in the number of vehicles. Traffic congestion appears when too many vehicles attempt to use a common transportation infrastructure with limited capacity. To reduce traffic congestion, several methods have been proposed, such as time Series, Kalman Filtering, neural networks, state space reconstruction, and non-parametric regression. Some of these have been successful at practical or simulated forecasting (Zheng, et. al., 2009). However, due to the complexity of traffic flow and the properties of forecasting methods, it is very difficult to achieve accurate forecast results using only one method or model. Some model parameters need to be determined before the model can be used to forecast the traffic volume, and the structure of the model depends on these parameters, which are computed 
from the historical and real time traffic data in practice. For a successful forecast of traffic flow, the model needs to perceive variations in the environment and adjust the parameters automatically. Furthermore, it is important that the forecast model take into consideration abnormal conditions that occur in real-time (Zhang et. al., 2003). The ability to accurately forecast future link times in the transportation network is a critical of component for many transportation system applications. Travel time in an urban traffic environment is highly time-dependant due to random fluctuations in travel demands, interruptions caused by traffic control devices, road incidents, and weather conditions. It has been increasingly recognized that for many transportation applications, estimates of the mean and variance in travel times significantly affect the accuracy of forecasting approaches. Figure 1 compares the travel data collection based on cellular systems to data collection based on sensors. The current system supports a data resolution of 2.5 minutes.

\section{Concept}

To overcome the lack of an ITS and the GNSS, we propose a hybrid scheme for travel forecasting based on real-time data, as illustrated Figure 1. Travel data collected via mobile services, and is controlled by satellites. Because some urban areas cannot be covered by cellular systems, we use satellite services (GPS/ GNSS) (Berzina et.al. 2014) (Hong et. al., 2014) to calculate travel time data. The major advantage of using mobile agents lies in their ability to move from one place to another and carry out their tasks autonomously, to manage dynamic negotiations with other mobile agents, to draw conclusions from plausible reasoning after updating their local information, and to develop themselves in the future in ways that are appropriate for newly implemented services in the system, in order to deal with their affiliated Quality of Experience (QoE). The mobile agent approach has been used in dynamic systems in many fields and has been reported in the literature. A mobile agent interacts autonomously to provide solutions. In this paper, mobile agents are incorporated into intelligent transportation systems in order to improve dynamic traffic management. A mobile agent is an actively communicating, cooperative, entity in a dynamic environment, which plays a role in updating travel data. The mobile agent adds new performance to the concept by perceiving the environment, which is needed for dynamic traffic data management. Its task is to sense and monitor the state of the traffic flow through the road network in order to enable better usage of the network, by updates traffic data. Middleware is deployed to monitor the traffic flow and speed variations through road network based on the QoE of end users.

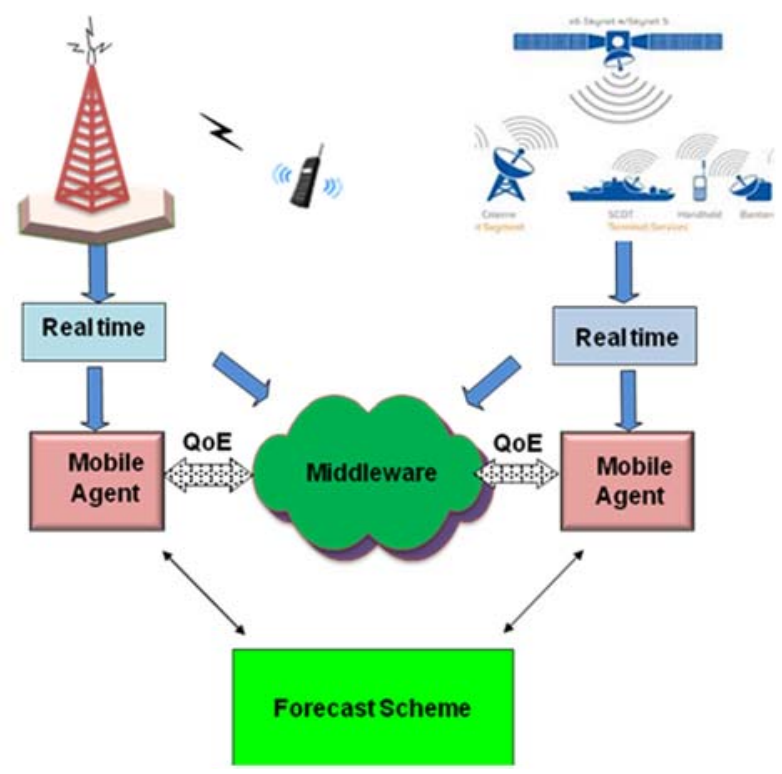

Figure 1. Forecasting model

\section{Short- Term Forecast Model}

\subsection{Methodology}

In this section, we introduce a forecasting model based on the moving average. There are three types of moving average: the simple moving average (SMA), the weighted moving average (WMA), and the 
exponential moving average (EMA). In this study, an exponential moving average was used. This form of average uses a weighting or a smoothing factor that decreases exponentially (Andrada-Felix et. al., 2008). The weighting for each older data point decreasing exponentially, gives much more importance to recent observations, while not discarding the older observations entirely. The forecast model is divided into two phases: detection phase, and forecasting phase. The detection phase focuses on an analysis of collected data. To increase accuracy in this phase we have to detect abnormal events in the collected data. The forecasting phase is based on the exponential moving average. The robustness and accuracy of the exponential smoothing forecast are impressive. The accuracy of this technique depends on the weight smoothed alpha factor value of the current demand. To determine the optimal alpha factor value, we use a fitting curve.

There are two kinds of exponential moving average forecasting (EMA): one uses exponential moving average based historical information (EMA-H); the other uses exponential moving average based real-time information (EMA-R). The EMA-R possesses two main phases, namely, a detection phase and a forecast phase.

\subsection{Short-term forecasting based on historical Information}

The historical database is a collection of past travel observations of the system. The exponential smoothing forecasting method gives weight to the observed time series unequally. This is accomplished by using one or more smoothing parameters, which determine how much weight is given to each observation. The major advantage of exponential smoothing method is that it gives good forecasts in a wide variety of applications. In addition, the data storage and computing requirements are minimal, which makes exponential smoothing suitable for real-time forecasting.

$t t(t+1, k)=\alpha * t t^{M}(t, k)+(1-\alpha) * t t^{H}(t, k)$

where $0<\alpha \leq 1, \mathrm{tt}^{\mathrm{M}}(\mathrm{t}, \mathrm{k})$ is the actual travel time in section $k$ at time $t$, and $\mathrm{tt}^{\mathrm{H}}(\mathrm{t}, \mathrm{k})$ is the historical travel time in section $k$ at time $t$.

\subsection{Smoothing parameter alpha}

To achieve short-term traffic flow forecasting with high accuracy, the proposed forecast scheme called for optimization of the smoothing parameter alpha. Alpha determines how responsive a forecast is to sudden jumps and drops. It is the percentage weight given to the prior historical period, and the remainder is distributed to the other historical periods. Alpha is used in all exponential smoothing methods. The lower the value of alpha, the less responsive the forecast is to sudden change. The smoothing parameter "alpha" lies between 0 and 1. To determine the optimal smoothing factor, a sum of the square errors between the observed and the forecast alpha dose rates was analyzed by increasing the smoothing filter factor from 0.1 . The sum of the square errors decreases as the smoothing filter factor increases, as shown in Figure 2.

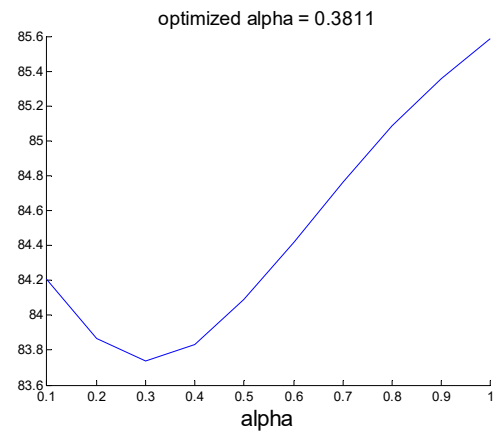

Figure 2. Smoothing parameter alpha

\subsection{Short term forecasting based on real-time information}

The occurrence of abnormal conditions in traffic flow travel information decreases of forecasts based on historical information and may increase the complexity of the forecasting of unusual incidents. The forecasting model based on real-time information gives a little weight to historical information and great weight to real-time observations. 
$t t(t+1, k)=t t^{H}(t+1, k)+\gamma *\left(t t^{M}(t, k)-t t^{H}(t, k)\right)$

where $0<\gamma<1$

\subsection{Smoothing parameter -gamma}

Figure 3 shows that the value of gamma for real-time forecasting is close to 0.9885 .

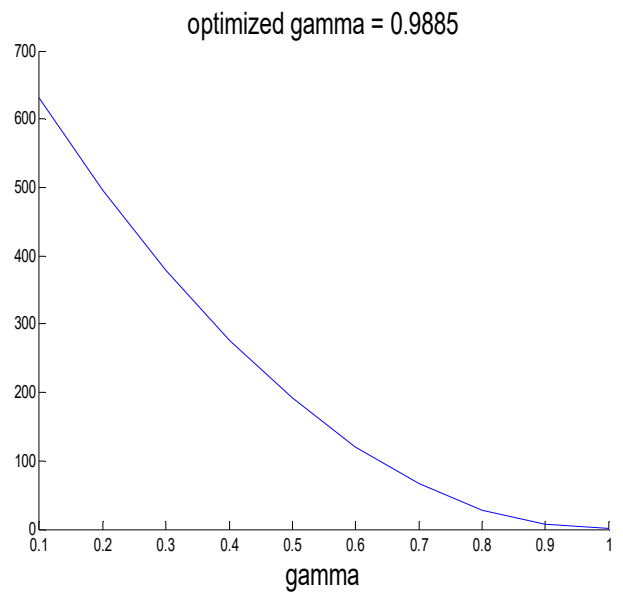

Figure 3. Smoothing parameter gamma

\subsection{Heterogeneous Road Network Management}

This concept can be adapted for managing various heterogeneous road network traffic, such as urban road traffic and highway traffic whose mean speed vary. The concept can be used also for various road networks worldwide. The management of heterogeneous road networks involves multiple lane roads, traffic positioning determination, vehicle location estimation, and travel forecasting. Various methods have been proposed for estimating the positions of vehicles, such as the use of wi-fi, cellular networks, sensors, and navigation systems. Nowadays, navigation systems such as the GNSS and GPSs are used widely for vehicle position detection. Navigation systems also provide travel information, destination directions, road maps, real-time road conditions, and vehicle speeds. Management of a heterogeneous road network required locating the vehicle within a road network. Navigation satellite systems, wi-fi, cellular systems, and sensors are used to accomplish this. However, their results are often inaccurate. To improve the detection of vehicle position within a road network, we propose a map- matching (MM) method, which is used often to obtain the real- time positions of vehicles in a road network. In basic terms, map-matching considers a trajectory $T$ and a road network $N$, and travel time $T$. The method detects newly arriving vehicle in section $A \in N$, through which $T$ is believed to have traveled in time $t$ and then allocates a precise position along A to which $T(t)$ should be matched. This method aims to identify the correct road segments and to determine the vehicle location on that segment (Quddus et, al., 2007).

Various map-matching methods have been proposed. Quddus et al. (2007) introduce a mapmatching strategy based on distance and orientation, which does not involve any further knowledge about the movement besides the position samples. Civilis et al. introduced a map-matching algorithm based on edge distance and direction, like that of Quddus et al. (2007), for updating location by tracking the users of location-based services. (Yin and Wolfson, 2004) proposed an algorithm based on a weighted graph representation of the road network in which the weights of each edge represent the distance of the edge to the trajectory. The improved map- matching method that is proposed here uses an algorithm based on local path searching and enable better determination of vehicle position within a road network, as illustrated in Figure 4. The model starts by computing the short- term travel forecast in real- time for all of the segments of a road network. When a vehicle demands service, the map-matching system detects its position in the road network. The heterogeneous road management model introduces the optimal path (start point to destination point) by considering the adaptive mean speed for each segment of the path. 


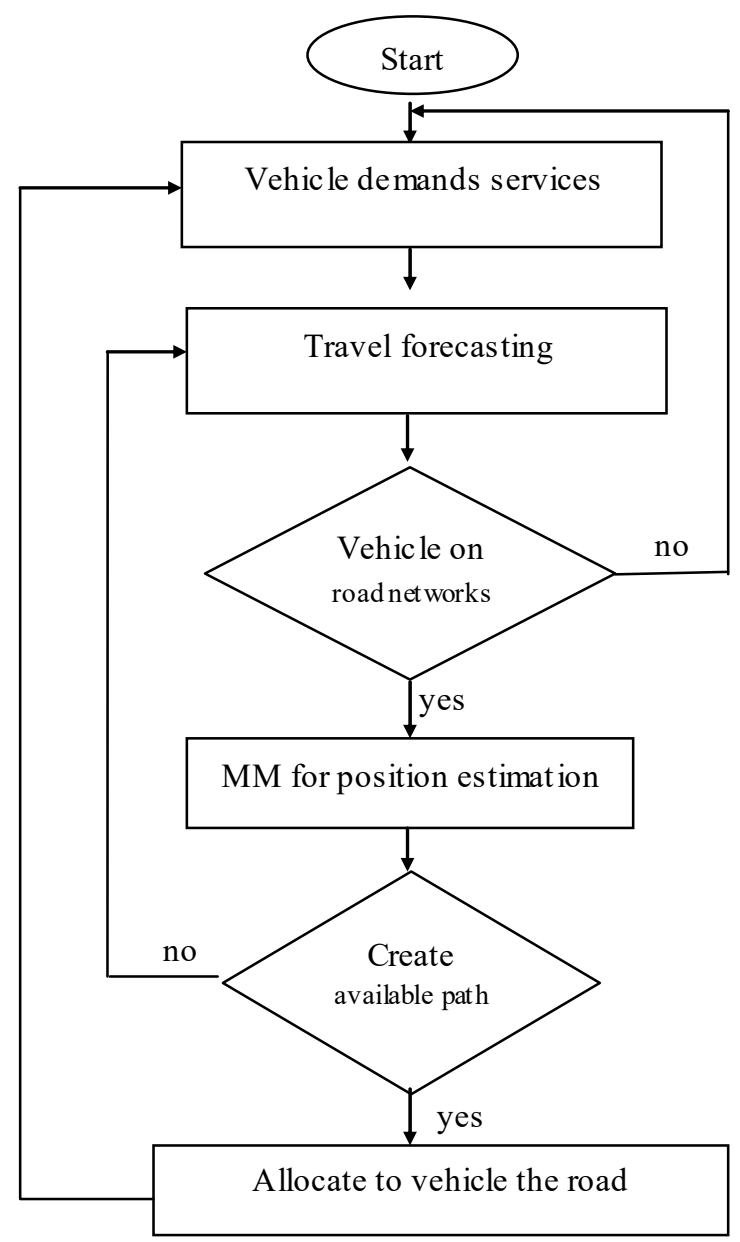

Figure 4. Road traffic management

\section{Evaluation of Forecast Model}

\subsection{Simulation}

We used the MATLAB environment to process the travel data. Figure 5 shows a comparison of travel data collected via mobile services with travel data collected by sensors. The current system supports a data resolution of 2.5 minutes. Figure 6 compared the EMA to the optimized EMA based on historical observations (travel data) (Raiyn et. al. 2014). Figures 7 and 8 present the actual observations compared to the EMA and to the EMA based on real-time information, respectively. The results indicate that all three moving average methods had more or less similar performance in forecasting short-term travel times. However, as one would expect the method using optimized weights produced slightly better forecasts at a higher computational cost. The quality of the forecasts diminished as the time for which the forecasts were made was projected farther into the future. Moving average methods overestimate travel speeds during slow-downs and underestimate them when traffic congestion is clearing up and speeds are increasing. Figure 8 presents a comparison between the exponential moving average- based historical information (EMA-H) and the exponential moving average based real-time information (EMA-R) compared to actual observations. The EMA-H detects abnormal conditions in traffic flow based on pervious data collected in the same location at the corresponding time. The advantage of the EMA-H is its ability to identify incident traffic flow. However, an incident occurring with the same characteristics in the future is not certain. Figure 8 demonstrates that the EMA-R can identify any incident in the traffic flow and provide incident clearness. Figure 9 compares the SMA, WMA, and EMA based on root mean squared error (RMSE). 


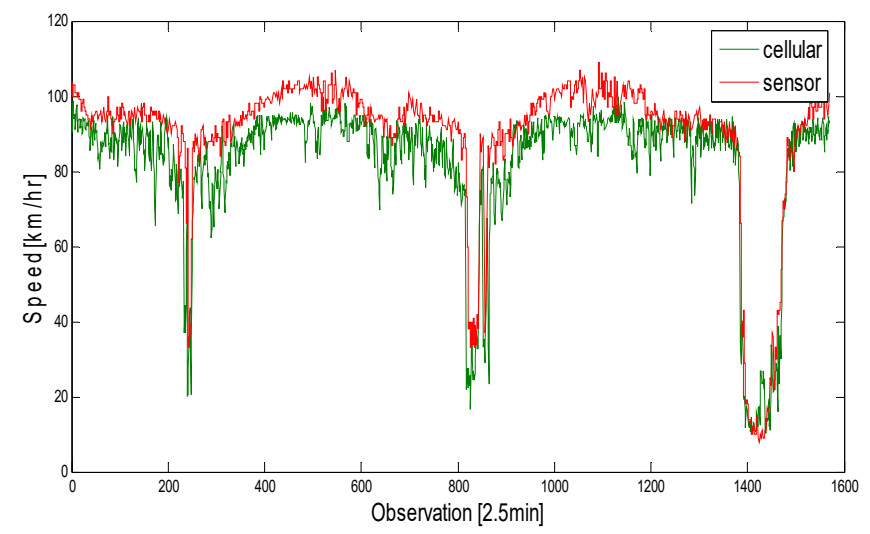

Figure 5. Cellular versus sensor travel data

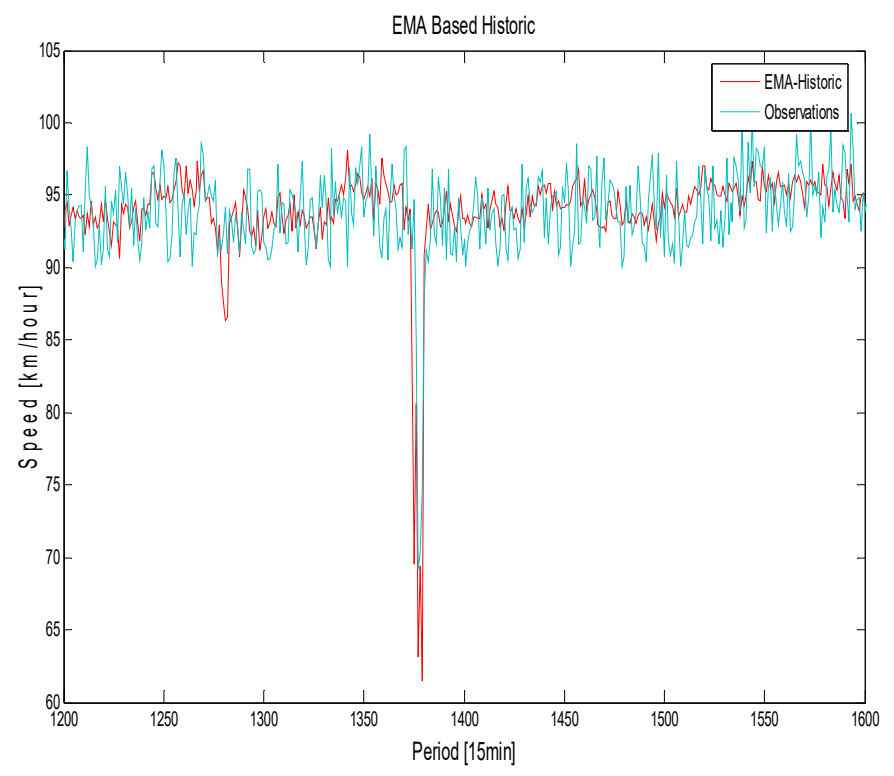

Figure 6. EMA-H versus actual observations

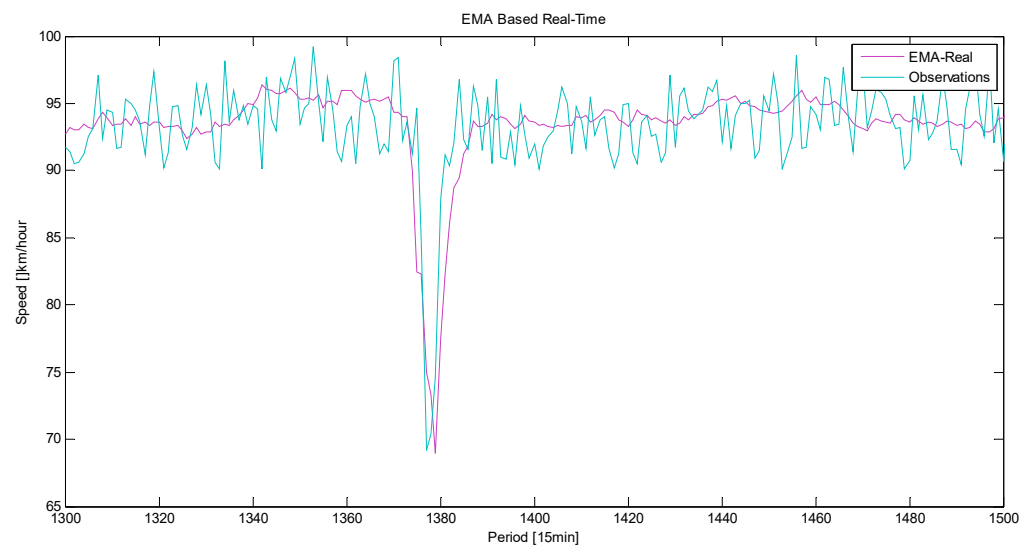

Figure 7. EMA-R versus actual observations 


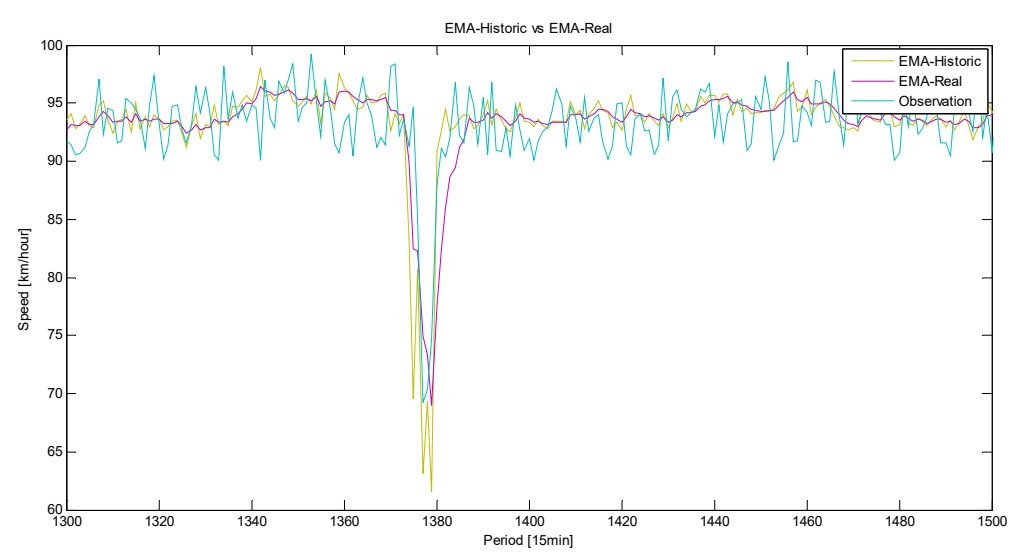

Figure 8. EMA-H and EMA-R compared

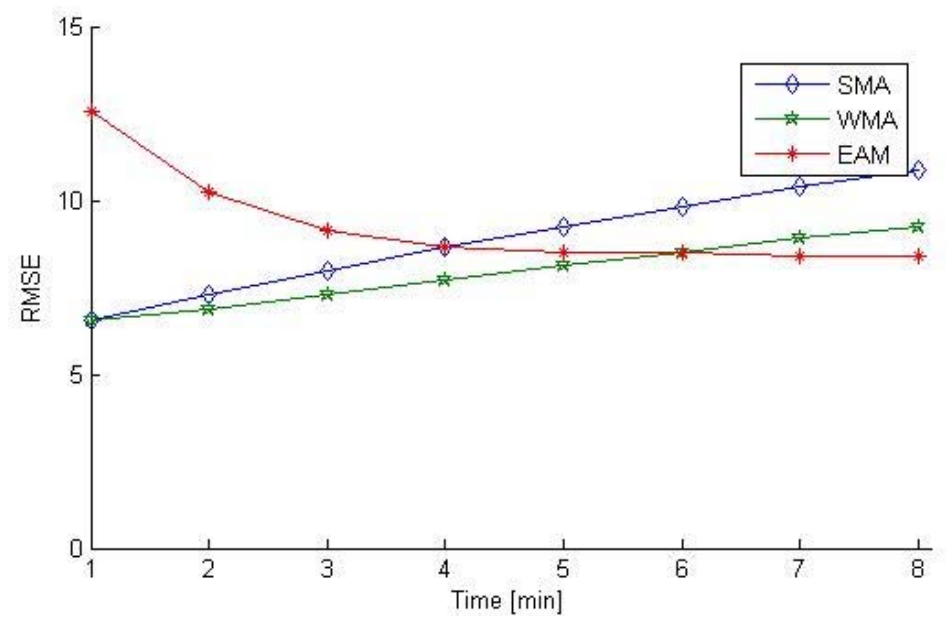

Figure 9. Root Mean Squared Error

\subsection{Position based speed}

The traffic management system also involves use of a geographic information system (GIS) to provide a digital map of the road network. It divides the high-way into sections (of 300 meters) and computes the speed for each section. The travel forecast for each section is adapted to traffic load and weather conditions. For speed indications on the digital map, we have used keyhole markup language (KML). KML is an XML notation for geographic annotation and visualization within Internet-based, twodimensional maps and three-dimensional Earth browsers. KML was developed for use with Google Earth, which was originally named Keyhole Earth Viewer. It was created by Keyhole, Inc, which was acquired by Google in 2004. Here is an example of a KML file which describes a point (longitude $=-1.82675$, latitude $=51.179045$, altitude $=0$ ):

$<$ ?xml version="1.0" encoding="UTF-8"? >

$<\mathrm{kml}$ xmlns="http://earth.google.com/kml/2.2" $>$

$<$ Placemark $>$

$<$ name $>$ Simple placemark $</$ name $>$

$<$ Point $>$

$<$ coordinates $>-1.82675,51.179045,0<$ coordinates $>$

$<$ /Point $>$

$<$ Placemark $>$

$</ \mathrm{kml}>$ 
Geographic features can be represented by several lines of KML code because a KML file is textformatted. Layers are an important feature of Google Earth, which provide access to all kinds of other data. All sorts of information, including videos, photos, real-time weather, 3D images of buildings, and more can be presented in KML and displayed in different layers. Figure 11 shows speed based locations on the highway between Haifa and Tel Aviv. Table 1 presents travel data forecast based on the SMA scheme. Figure 10 illustrates the travel speed averages related to different road sections. Figures 11, 12, and 13 show the travel speeds based on position. The road has been divided in 300-meter-long sections, and the Travel speed has been assigned to each section.

Table 1. Estimated travel speed average

\begin{tabular}{|l|c|c|c|c|c|}
\hline Sections & 0 & 1 & 2 & 3 & 4 \\
\hline $\begin{array}{l}\text { Normal Mean } \\
\text { km/hour) }\end{array}$ & 53.8606 & 47.5177 & 45.3111 & 44.7848 & 47.6886 \\
\hline Sections & 5 & 6 & 7 & 62.9577 & 75.2875 \\
\hline $\begin{array}{l}\text { Normal Mean } \\
\text { (km/hour) }\end{array}$ & 58.1212 & 59.7911 & 12 & 13 & $14-16$ \\
\hline Sections & 10 & 11 & 78.8352 & 81.3669 & 81.1209 \\
\hline $\begin{array}{l}\text { Normal Mean } \\
\text { (km/hour) }\end{array}$ & 76.9951 & 77.2779 & & \\
\hline
\end{tabular}

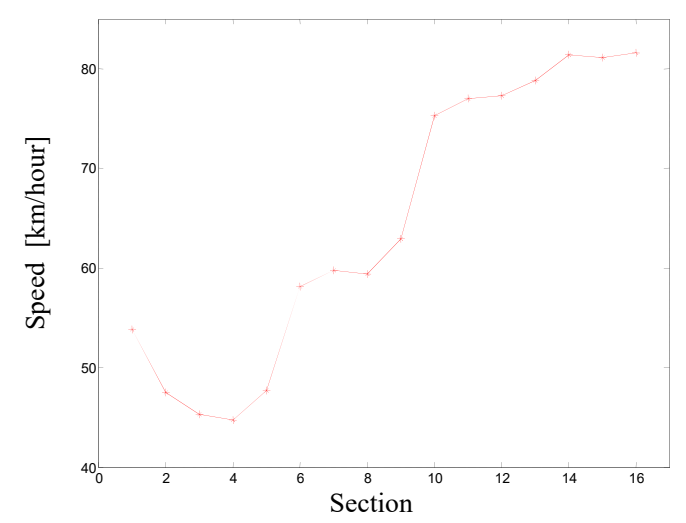

Figure 10. Estimated travel speed average

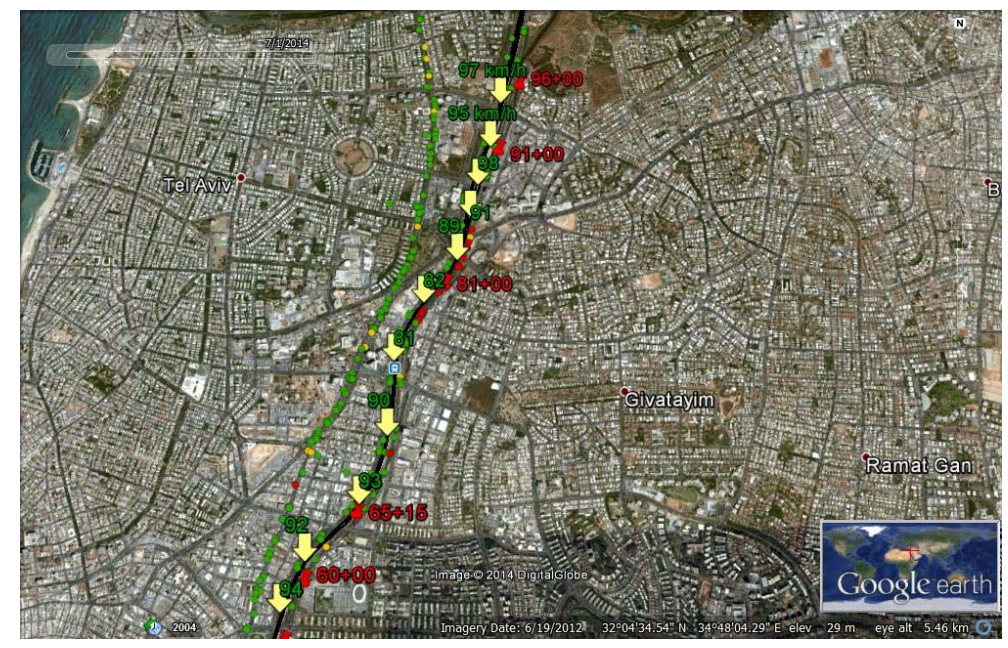

Figure 11. Traffic flow 


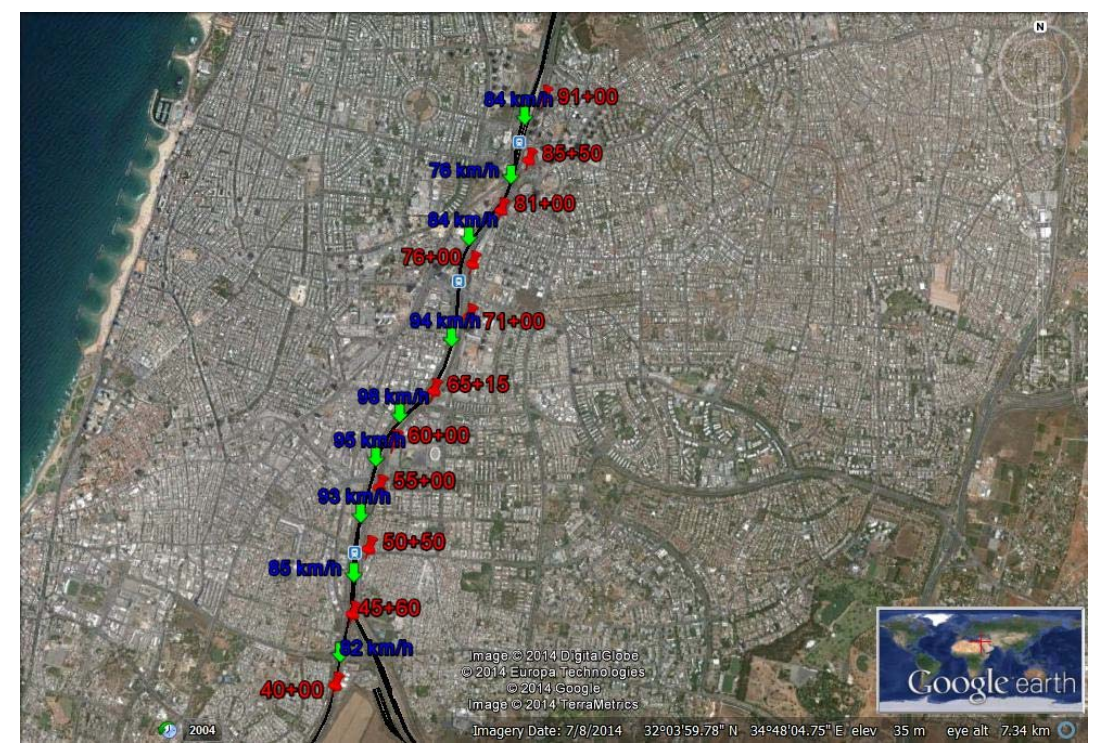

Figure 12. Travel speed per section

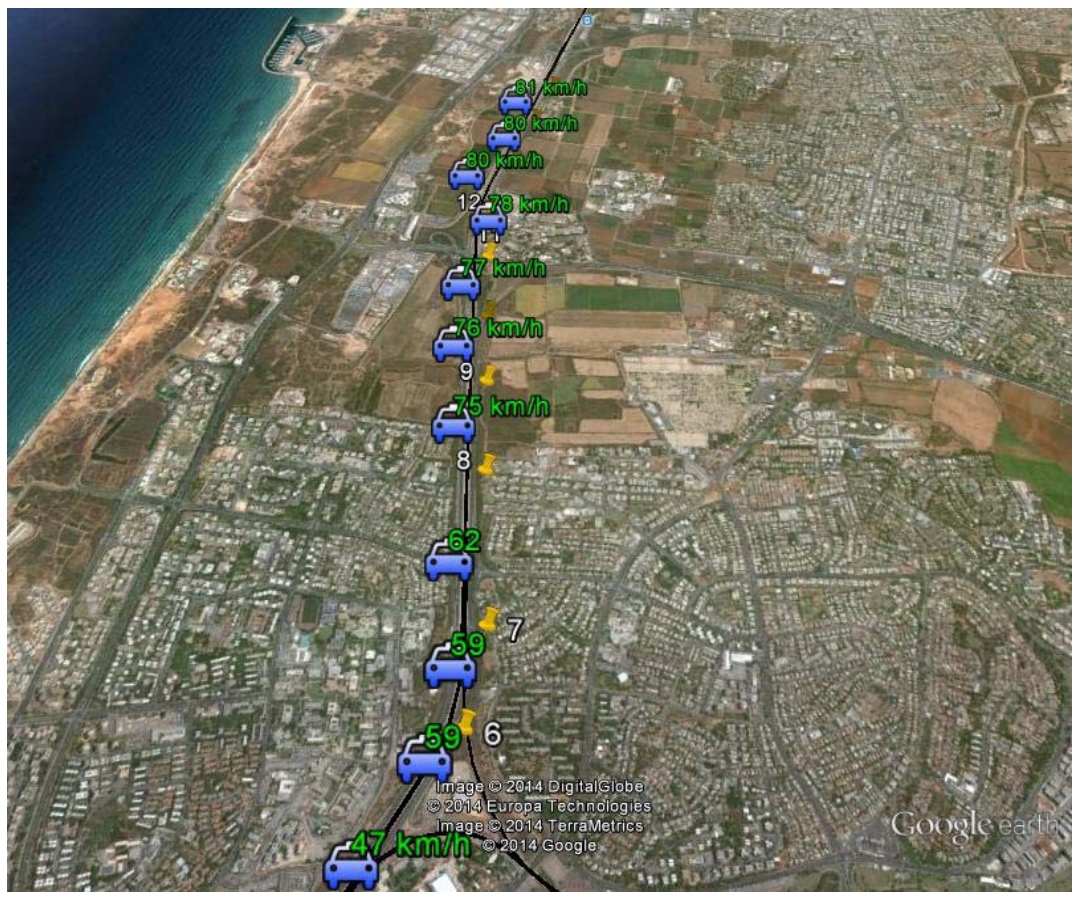

Figure 13. Location based speed

\section{Conclusion and future works}

In this paper we have discussed travel data management. We have introduced three kinds of moving average schemes, simple moving average (SMA), weighted moving average (WMA), and exponential moving average (EMA). The exponential moving average scheme offered the best results compared to other forecast schemes, furthermore EMA is the most accurate method. The exponential moving average is used for real short- term travel forecast. The travel data collection is based on cellular phone service. Due to a lack of urban coverage in cellular systems and in the GNSS, we have introduced a hybrid scheme that is based on both cellular systems and the GNSS data. GNSS data are used to determine the floating data positioning in urban area. Future work will consider the management of travel Big Data for heterogeneous road networks with dynamic traffic conditions. 


\section{References}

1. Andrada-Felix, J. and Fernandez-Rodriguez, F. (2008) Improving Moving Average Trading Rules with Boosting and Statistical Learning Methods, Journal of Forecasting, 27, pp.433-449.

2. Berzina, L., Faghri, A., Shourijeh, M.T. and Li, M. (2014) Developing of a Post-Processing Automation Procedure for the GPS-Based Travel Time Data Collection Technique, Journal of Transportation Technologies, 4, pp. 63-71.

3. Borzacchielo, T.M. (2010) The use of data from mobile phone networks for transportation applications, TRB 2010 Annual Meeting.

4. Elvik, R. (2000) How much do road accidents cost the national economy?, Accident Analysis and Prevention 32, pp.849-851.

5. Hong, W., Choi, K., Lee, E., Im, M. and Heo, M. (2014) Analysis of GNSS Performance Index Using Feature Points of Sky-View Image, IEEE Transactions on Intelligent Transport Systems, vol. 15, no. 2, pp.889-895.

6. Quddus, M.A., Ochieng, W.Y. and Noland, R.B. (2007) Current map-matching algorithms for transport applications: State-of-the art and future research directions, Transportation Research Part C 15, (2007), pp. 312-328

7. Raiyn, J. and Toledo, T. (2014) Real-Time Short-Term Forecasting Based on Information Management, Journal of Transportation Technologies, 4, 11-21.

8. Tu, H., Van Lint, H. and Van Zuylen, H. (2008) The Effects of Traffic Accidents on Travel Time Reliability, IEEE Conference on Intelligent Transportation Systems, Beijing, China, October 12-15.

9. Velaga, N.R., Quddus, M.A. and Bristow, A.L. (2010) Detecting and Correcting Mapmatching Errors in Location-Based Intelligent Transport Systems, 12th WCTR, July 11-15, Lisbon, Portugal.

10. Yin, H. and Wolfson, O. (2004) A Weight-Based Map Matching Method in Moving Objects Databases, In Proceedings of 16th SSDBM conference, Santorini Island, Greece, pp. 437-438.

11. Zhang, X., Rice, J.A. (2003) Short-term travel time prediction. Transport. Res. Part C: Emer. Technol. 11 (3-4), pp.187-210.

12. Zheng, X. and Liu, M. (2009) An Overview of Accident Forecasting Methodologies. Journal of Loss Prevention in the Process Industries, 22. pp. 484-491. 